\title{
Gelatin-Modified Biomimetic Apatite Coatings
}

\author{
Barbara Bracci ${ }^{1}$, Paola Torricelli ${ }^{2}$, Silvia Panzavolta ${ }^{1 *}$, Katia Rubini ${ }^{1}$, Milena Fini ${ }^{2}$, Adriana Bigi ${ }^{1}$ \\ ${ }^{1}$ Department of Chemistry “G. Ciamician”, University of Bologna, Bologna, Italy; ${ }^{2}$ Preclinical and Surgical Studies Laboratory, Re- \\ search Institute Codivilla Putti-Rizzoli Orthopaedic Institute, Bologna, Italy. \\ Email: *silvia.panzavolta@unibo.it
}

Received January $8^{\text {th }}, 2012$; revised February $18^{\text {th }}, 2012$; accepted March $4^{\text {th }}, 2012$

\begin{abstract}
Biomimetic coatings constituted of gelatin and nanocrystalline apatite were deposited on titanium substrates from a slightly supersaturated calcium phosphate $(\mathrm{CaP})$ solution enriched with different amounts of gelatin. Although the biopolymer inhibits the crystallization of the inorganic phase, as shown by the reduction of the mean dimensions of the spherical aggregates and of the degree of crystallinity of the apatitic phase on increasing gelatin concentration, the deposition of a uniform layer of nanocrystalline apatite takes place in a few hours. Gelatin incorporation into the precipitate increases on increasing its concentration in solution, up to about $20 \mathrm{wt} \%$. Osteoblast-like MG63 cells cultured on the coatings display good proliferation and increased values of the differentiation parameters with respect to the control. The presence of gelatin improves significantly the biological response to the biomimetic coatings, as shown by the higher values of proliferation, collagen type I and osteocalcin production, as well as alkaline phosphatase activity stimulation.
\end{abstract}

Keywords: Biomimetic Coating; Gelatin; Hydroxyapatite

\section{Introduction}

The presence of a thin layer of bone-like calcium phosphate on metallic implants for bone substitution at high load-bearing sites enhances fixation stability and promotes integration with the host tissue, thanks to the excellent bioactivity of calcium phosphates. A number of different methods can be utilized to deposit the coating [1-3]. The biomimetic approach involves the use of slightly supersaturated solutions at $\mathrm{pH}$ and temperature values similar to those characteristic of the biological fluids [4]. Starting from the very first formulation of Simulated Body Fluid (SBF) [5], which mimics the ionic composition of blood plasma, a number of different solutions have been proposed [4,6-8]. The main advantages of the biomimetic method include low cost, deposition of an apatitic phase similar to the poor crystalline carbonated apatite of bone, possible application to complexshaped and porous materials, possible co-precipitation of ions, drugs, macromolecules and biological molecules together with the calcium phosphate [9-13]. In particular, the possibility to improve the biological performance of the coating by co-precipitating proteins, including bovine serum albumin, bone morphogenetic protein, and collagen, has been successfully explored [14-20]. The interest towards collagen obviously arises because of its relevant

${ }^{*}$ Corresponding author. presence in the organic matrix of bone where the deposition of inorganic deposits occurs. In order to reduce possible immunogenic reaction and improve processability, recombinant human-like collagen has been employed too [21]. Gelatin is obtained by thermal denaturation or physical and chemical degradation of collagen through the breaking of the triple-helix structure into random coils [22]. With respect to collagen, gelatin does not express antigenicity in physiological conditions, it is completely resorbable in vivo and its physico-chemical properties can be suitably modulated; furthermore it is much cheaper and easier to obtain in concentrate solutions [23]. For these reasons gelatin is widely used in pharmaceutical and medical fields for a variety of applications including tissue engineering, wound dressing, drug delivery and gene therapy [24]. In this work we studied the possibility to co-precipitate gelatin together with nanocrystalline apatite. To this aim, we utilized a slightly supersaturated solution $(\mathrm{CaP})$, previously developed by our group, which displays a quite simple ionic composition and allows to get a uniform nanocrystalline apatite coating in a few hours $[6,25]$. Herein we report the results of the chemical, structural and morphological investigation carried out on the coatings deposited in the presence of different concentrations of gelatin, together with the results of in vitro tests that demonstrate the beneficial effect of gelatin on osteoblast proliferation and differentiation. 


\section{Materials and Methods}

\subsection{CaP Calcifying Solutions}

The slightly supersaturated $\mathrm{CaP}$ solution was prepared as previously reported [6]. Briefly, the reagent grade chemical $\mathrm{CaCl}_{2} \cdot 2 \mathrm{H}_{2} \mathrm{O}$ was dissolved in double distilled water and buffered at $\mathrm{pH} 7.2$ with Hepes (Ca solution). The reagent grade chemicals $\mathrm{Na}_{3} \mathrm{PO}_{4} \cdot 12 \mathrm{H}_{2} \mathrm{O}$, and $\mathrm{NaHCO}_{3}$ were dissolved in double distilled water and buffered at pH 7.2 with Hepes (P solution). CaP supersaturated calcifying solution was freshly prepared by mixing $500 \mathrm{ml}$ of the Ca solution and $500 \mathrm{ml}$ of the $\mathrm{P}$ solution at $37^{\circ} \mathrm{C}$. The final ionic concentration of the $\mathrm{CaP}$ solution was 2.5 $\mathrm{mM} \mathrm{Ca}^{2+}, 2.5 \mathrm{mM} \mathrm{PO}_{4}^{3-}$, and $18 \mathrm{mM} \mathrm{HCO}_{3}^{-}$.

Type A gelatin (280 Bloom, Italgelatine S.p.A.) from pig skin was used for the preparation of gelatin modified $\mathrm{CaP}$ solutions. $\mathrm{CaP}$ solutions containing increasing amounts of gelatin, from 0.05 to $1 \mathrm{wt} \%$, were prepared, by dissolving gelatin powder in the buffered solution.

\subsection{Powder Samples}

In order to better evaluate the effect of gelatin on the inorganic phase precipitated from the calcifying solution, preliminary data were obtained on the powders precipitated in the absence of Ti substrates: after mixing the cationic solution with the anionic solution the temperature was kept at $37^{\circ} \mathrm{C}$ for 6 hours, then the precipitate deposited on the bottom of the flask was filtered, washed with double distilled water and dried at $37^{\circ} \mathrm{C}$ overnight.

In the following, the powder samples are labelled as in Table 1.

X-ray diffraction (XRD) analysis was carried out by means of a PANalytical powder diffractometer equipped with a fast $\mathrm{X}^{\prime}$ Celerator detector. CuKa radiation was used $(40 \mathrm{~mA}, 40 \mathrm{kV})$. The $2 \theta$ range was from $3^{\circ}$ to $50^{\circ}$ with a step size of $0.0334^{\circ}$ and a counting time of $19 \mathrm{~s}$. In order to evaluate the coherence lengths of the crystals, further X-ray powder data were collected in the relevant regions of $2 \theta$, from $24.5^{\circ}$ to $27.5^{\circ}$, with a step size of $0.334^{\circ}$ and a counting time of $1000 \mathrm{~s}$.

For infrared adsorption (FT-IR) analysis, $1 \mathrm{mg}$ of the ground samples was carefully mixed with $300 \mathrm{mg}$ of $\mathrm{KBr}$ (infrared grade) and pelletized under a pressure of 10 tons for $2 \mathrm{~min}$. The pellets were analyzed using a Nicolet FT 205 IR spectrophotometer to collect 32 scans in the range $4000-400 \mathrm{~cm}^{-1}$ at a resolution of $4 \mathrm{~cm}^{-1}$.

Morphological investigation was performed using a Philips XL-20 scanning electron microscope operating at $15 \mathrm{kV}$. The samples were sputter-coated with gold before examination.

Gelatin content of the powders was determined through thermogravimetric analysis, which was carried out using a Perkin Elmer TGA-7. Heating was performed in a platinum crucible in air flow $(20 \mathrm{~mL} / \mathrm{min})$ at a rate of $10^{\circ} \mathrm{C} / \mathrm{min}$ up to $800^{\circ} \mathrm{C}$. The samples weights were in the range $5-10 \mathrm{mg}$.

\subsection{Substrate Coatings}

Disk-shaped Ti substrates $(\varnothing 12 \mathrm{~mm}$; thickness $1.5 \mathrm{~mm}$, SAMO SpA, Milan), mechanically polished and subsequently submitted to acid etching to get an extended active surface $[25,26]$, were rinsed in acetone, and then in double distilled water.

Coating of the substrates was performed by immersion of the etched disks in the CaP calcifying solution and, alternatively, in the gel-modified calcifying solutions at $37^{\circ} \mathrm{C}$ for $6 \mathrm{~h}$. Coated samples were accurately rinsed in double distilled water, and dried at $37^{\circ} \mathrm{C}$ overnight.

In the following, the coated titanium samples are labelled as in Table 1.

XRD measurements were performed on the coatings using a PANalytical powder diffractometer equipped with a fast $\mathrm{X}^{\prime}$ Celerator detector. CuKa radiation was used $(40 \mathrm{~mA}, 40 \mathrm{kV})$. The $2 \theta$ range was from $24^{\circ}$ to $34^{\circ}$ with a step size of $0.068^{\circ}$ and a counting time of $1500 \mathrm{~s}$.

Morphological investigations of the coatings were performed using a Philips XL-20 scanning electron microscope, operating at $15 \mathrm{kV}$. The samples were sputtercoated with gold before examination.

In order to appreciate the presence of gelatin in the coatings, we performed microscopy fluorescence observations on samples obtained by adding Fluorescein isothiocyanate (FITC) to the gelatin-containing calcifying solutions, at the concentration of $0.1 \%$ respect to gelatin weight.

Table 1. Gelatin contents of solid powders synthesized at increasing gelatin content in solution. The values are expressed as wt\%. The labels used for both the powder samples and the related coatings are reported.

\begin{tabular}{cccc}
\hline Gelatin concentration in solution & Gelatin concentration in the powders & Label of powder samples & Label of the coated samples \\
\hline- & - & $\mathrm{CaP}$ & $\mathrm{TiCaP}$ \\
0.05 & $4.9 \pm 0.1$ & $\mathrm{CaP} 0.05$ & $\mathrm{TiCaP} 0.05$ \\
0.1 & $6 \pm 1$ & $\mathrm{CaP} 0.1$ & $\mathrm{TiCaP} 0.1$ \\
0.5 & $14.5 \pm 0.5$ & $\mathrm{CaP} 0.5$ & $\mathrm{TiCaP} 0.5$ \\
1 & $19.5 \pm 0.4$ & $\mathrm{CaP} 1$ & $\mathrm{TiCaP} 1$ \\
\hline
\end{tabular}




\subsection{In Vitro Studies}

\subsubsection{Osteoblast Culture}

MG-63 human osteoblast-like cells were cultured in DMEM medium (Sigma, UK) supplemented with $10 \%$ FCS, and antibiotics $(100 \mathrm{U} / \mathrm{ml}$ penicillin, $100 \mu \mathrm{g} / \mathrm{ml}$ streptomycin). Cells were detached from culture flasks by trypsinization, and centrifuged. Cell number and viability were checked with trypan blue dye exclusion test.

MG-63 osteoblast-like cells were plated at a density of $2 \times 10^{4}$ cells $/ \mathrm{ml}$ in 24 -well plates containing six sterile samples for each biomaterial: titanium coated with $\mathrm{CaP}$ (TiCaP, reference), and $\mathrm{CaP}$ added with $0.1 \%$ or $0.5 \%$ gelatin (TiCaP0.1 and $\mathrm{TiCaPO} 0.5$ respectively). The same concentration of cells was seeded into empty wells as a control (CTR). The medium was changed with DMEM to which $\beta$-Glycerophosphate $\left(10^{-2} \mathrm{M}\right)$ and Ascorbic acid $(50 \mu \mathrm{g} / \mathrm{ml})$ were added to activate the osteoblasts. Plates were cultured under standard conditions, at $37^{\circ} \mathrm{C} \pm 0.5^{\circ} \mathrm{C}$ with $95 \%$ humidity and $5 \% \mathrm{CO}_{2} \pm 0.2$ up to 7 days. For the production of osteocalcin the culture medium was enriched with $1,25(\mathrm{OH})_{2} \mathrm{D}_{3}\left(10^{-9} \mathrm{M}\right) 48$ hours before end of each experimental time.

\subsubsection{Osteoblast Proliferation}

Cell proliferation and viability (1, 3, and 7 days) was assessed by WST1 (WST1, Roche Diagnostics GmbH, Manheim, Germany) colorimetric reagent test. This viability test is based on the reduction of a tetrazolium salt to a soluble formazan salt by a reductase of the mitochondrial respiratory chain that is active only in viable cells. $50 \mu \mathrm{l}$ of WST1 solution and $450 \mu \mathrm{l}$ of medium (final dilution: 1:10) were added to the cell monolayer, and the multi-well plates were incubated at $37^{\circ} \mathrm{C}$ for the next $4 \mathrm{~h}$. Supernatants were quantified spectrophotometrically at $450 \mathrm{~nm}$ with a reference wavelength of $625 \mathrm{~nm}$. Results of WST1 are reported as optical density (OD) and can be directly correlated with the cell number.

Phalloidin staining (specific for cellular microtubules) was performed after 24 hours from seeding to assess cell adhesion and colonization of samples: samples were washed in PBS and fixed in a solution of $4 \%$ formaldehyde in PBS for $15 \mathrm{~min}$ at $37^{\circ} \mathrm{C}$; then the samples were permeabilized in $0.5 \%$ Triton $\mathrm{X}-100$ for $15 \mathrm{~min}$, washed in PBS, and a FITC-conjugate phalloidin solution (1:100 in PBS) was added and incubated for $30 \mathrm{~min}$ at $37^{\circ} \mathrm{C}$. After washing, samples were examined by fluorescence microscopy and the images were evaluated by a computerized image analysis system (Kontron KS 300 software, Kontron Electronic GmbH, Eching bei Munchen, Germany) to calculate the percent coverage of samples area by adhered cells.

\subsubsection{Osteoblast Activity and Differentiation}

At 3 and 7 days the supernatant was collected from all wells and centrifuged to remove particulates, if any. Aliquots of the supernatants were dispensed into Eppendorf tubes for storage at $-70^{\circ} \mathrm{C}$ and assayed for Alkaline Phosphatase activity (ALP, Ostase BAP immunoenzymatic assay, Immuno Diagnostic Systems, UK), Collagen Type I (COL I, immunoenzymatic assay kit, Uscn Life Sciences, UK), Osteocalcin (OC, enzyme Immunoassay kit, Bender MedSystems, Vienna, A), Transforming Growth Factor- $\beta 1$ and Interleukin 6, (TGF- $\beta 1$, IL-6, Quantikine Immunoassay, R\&D Systems, MN, USA). All the measured concentrations and activities were normalized by Total Protein content (TP, Total Protein micro-Lowry kit, SIGMA) to take into account the differences in cell growth.

\subsubsection{Cell Morphology}

Samples for each material, at the end of the experiment, were processed for Scanning Electron Microscopy (SEM): osteoblasts grown on the materials were fixed in $2.5 \%$ glutaraldehyde, in $\mathrm{pH} 7.4$ phosphate buffer $0.01 \mathrm{M}$ for $1 \mathrm{~h}$ and dehydrated in a graded ethanol series. After a passage in hexamethyldisilazane, the samples were air dried. The samples were sputter-coated with Au-Pd prior to examination with a Philips XL-20 Scanning Electron Microscope.

\subsubsection{Statistical Analysis}

Statistical evaluation of data was performed using the software package SPSS/PC ${ }^{+}$Statistics ${ }^{\mathrm{TM}} 10.1$ (SPSS Inc., Chicago, IL USA). Data are reported as mean \pm standard deviations (SD) of six replicate at a significance level of $\mathrm{p}<0.05$. After having verified normal distribution and homogeneity of variance, a one-way ANOVA was done for comparison between groups. The Scheffe's post hoc multiple comparison tests were performed to detect significant differences between groups. Pearson test was performed to assess correlation between the analyzed parameters.

\section{Results and Discussion}

The CaP solution can induce precipitation of a thin layer of poor crystalline hydroxyapatite on Ti substrates in just $1.5 \mathrm{~h}$ [25]. The thickness of the layer was previously found to increase with soaking time $[6,25]$. In this paper, in view of the possible inhibitory effect of gelatin on inorganic phase precipitation, the $\mathrm{Ti}$ substrates were maintained in the calcifying solutions for $6 \mathrm{~h}$. Accordingly, also the powders deposited from the calcifying solutions in the absence of the substrates were analyzed after the same period of time.

\subsection{Powder Samples}

In order to better evaluate the effect of gelatin on the 
inorganic phase precipitated from the calcifying solution, preliminary data were obtained on the powders precipitated in the absence of $\mathrm{Ti}$ substrates: after mixing the cationic solution with the anionic solution the temperature was kept at $37^{\circ} \mathrm{C}$ for 6 hours, then the precipitate deposited on the bottom of the flask was filtered, washed with double distilled water and dried at $37^{\circ} \mathrm{C}$ overnight.

The amount of gelatin incorporated in the inorganic phase was evaluated through thermogravimetric analysis. The thermogram of gelatin displays three thermal processes: the first process occurs between $25^{\circ} \mathrm{C}$ and $250^{\circ} \mathrm{C}$, and it is due to loss of water; the second one between $250^{\circ} \mathrm{C}$ and $450^{\circ} \mathrm{C}$ involves gelatin decomposition, and the third one between $450^{\circ} \mathrm{C}$ and $700^{\circ} \mathrm{C}$ corresponds to the combustion of the residual organic component [27]. Gelatin content of the precipitated powders evaluated from the TG/DTG plots (Figures 1(a)-(d)) increased on increasing gelatin concentration in the calcifying solution up to about $20 \mathrm{wt} \%$ (Table 1). The combustion process, which in pure gelatin occurs at about $600^{\circ} \mathrm{C}$, shifted at lower temperatures in the thermograms of the powders precipitated from $\mathrm{CaP}$ in the presence of gelatin. Moreover, the relative amount of weight loss associated to this peak with respect to the loss associated with the denaturation peak centered at $350^{\circ} \mathrm{C}$ was reduced with respect to that observed for pure gelatin. The TG/DTG plots reported in Figure 1 show that at relatively high protein content the combustion peaks is just a shoulder of the decomposition peak: the thermogram is similar to those characteristic of biologically calcified tissues, such as bone and tendons [27], and it indicates a strong interacttion between gelatin and the inorganic component during apatite formation. At variance, the TG/DTG plot recorded from a mechanical mixture of $20 \mathrm{wt} \%$ gelatin and $80 \mathrm{wt} \%$ poorly crystalline apatite (precipitated from $\mathrm{CaP}$ solution in the absence of gelatin) was quite similar to that of pure gelatin, in agreement with the lack of interaction between the two components of the mixture (Figure 2).

The X-ray diffraction patterns of the powders prepared in the presence of increasing amounts of gelatin were consistent with the presence of poorly crystalline hydroxyapatite as the unique crystalline phase (Figure 3 ). The broadening of the diffraction peaks increased on increasing gelatin content, in agreement with a progresssive reduction of the degree of crystallinity. The mean size of coherently scattering domains along the c-axis direction $\left(\tau_{002}\right)$ evaluated from the Scherrer equation, on the hypothesis of negligible microstrain, is reported in Table 2. $\tau_{002}$ values slightly decreased on increasing gelatin content, in agreement with the inhibitory role of gelatin on the crystallization of calcium phosphates [2830].

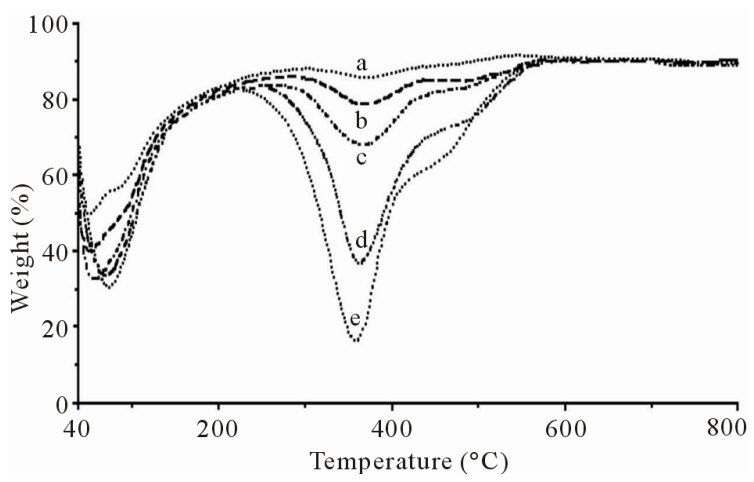

Figure 1. TG/DTG plots of the powders at different gelatin content. (a) CaP; (b) CaP0.05; (c) CaP0.1; (d) CaP0.5; (e) CaP1.

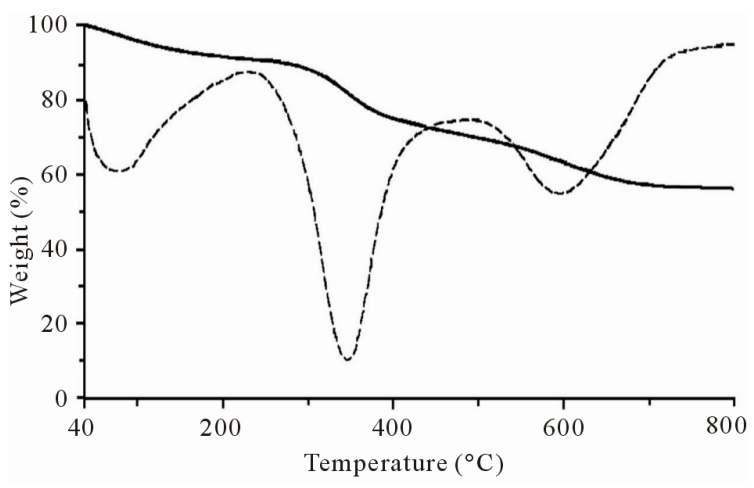

Figure 2. TG/DTG plot of the mechanical mixture of $\mathbf{2 0 \%}$ wt gelatin and $80 \%$ wt poorly cristalline apatite.

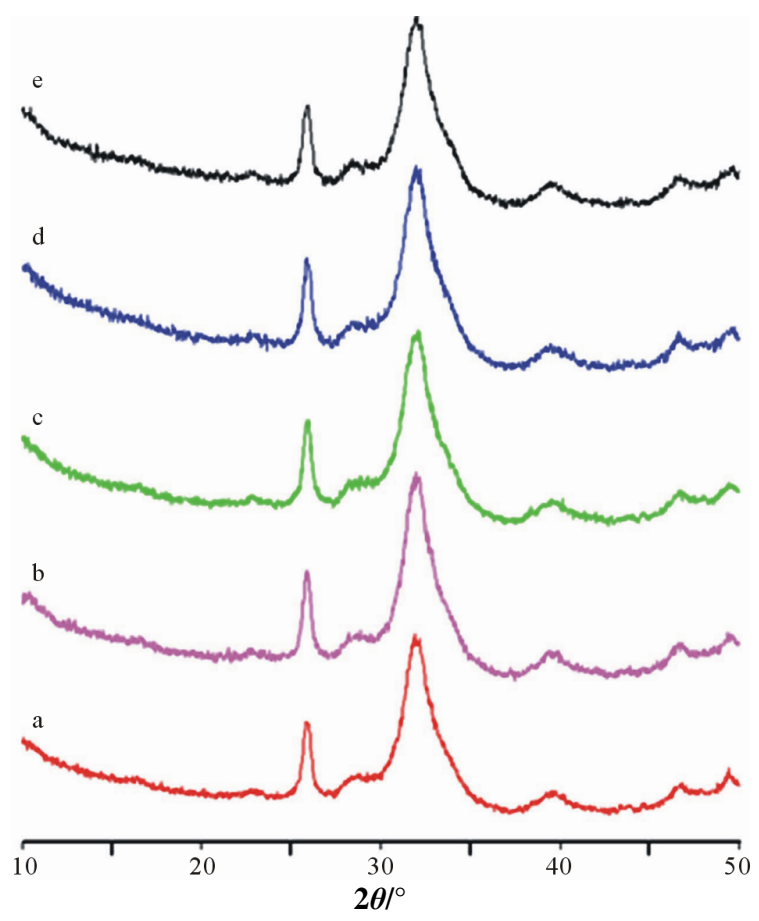

Figure 3. X-ray diffraction patterns of the powders prepared in the presence of increasing amounts of gelatin. (a) CaP; (b) CaP0.05; (c) CaP0.1; (d) CaP0.5; (e) CaP1. 
Electron microscope images showed that the CaP sample was constituted of almost spherical aggregates with mean diameter of about $2-4 \mu \mathrm{m}$ (Figure 4(a)). On increasing gelatin content, the mean dimensions of the spherical aggregates decreased to $1-2 \mu \mathrm{m}$, for the $\mathrm{CaPO} .1$ samples (Figure 4(c)), while the samples $\mathrm{CaP} 0.5$ and $\mathrm{CaP} 1$ appeared as almost uniform blocks (Figures 4(d) and (e)), where the single particles are barely detectable. (Figure 4(f)).

\subsection{Substrate Coatings}

Figure 5 reports the $\mathrm{X}$-ray diffraction patterns recorded from the Ti substrates after $6 \mathrm{~h}$ soaking in the different

Table 2. Coherent lengths $\left(\tau_{\mathrm{hkl}}\right)$ of the perfect crystalline domains, in the direction normal to 002 planes of the CaP powders, at increased gelatin content.

\begin{tabular}{cc}
\hline sample & $\tau_{002}(\mathrm{~nm})$ \\
\hline $\mathrm{CaP}$ & $30.1 \pm 0.4$ \\
$\mathrm{CaP} 0.05$ & $30.3 \pm 0.1$ \\
$\mathrm{CaP} 0.1$ & $29.4 \pm 0.4$ \\
$\mathrm{CaP} 0.5$ & $28.5 \pm 0.2$ \\
$\mathrm{CaP} 1$ & $28.7 \pm 0.3$ \\
\hline
\end{tabular}

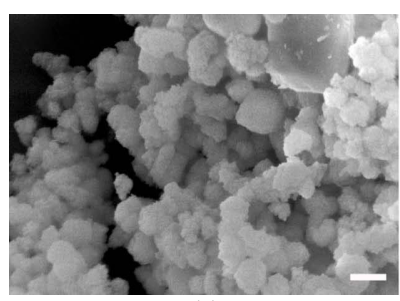

(a)

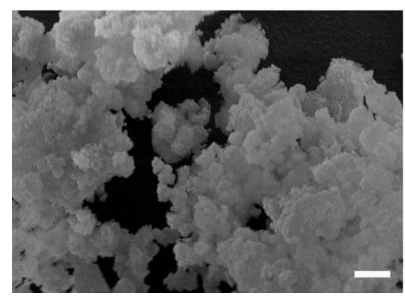

(c)

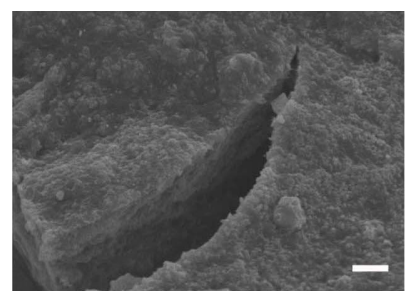

(e)

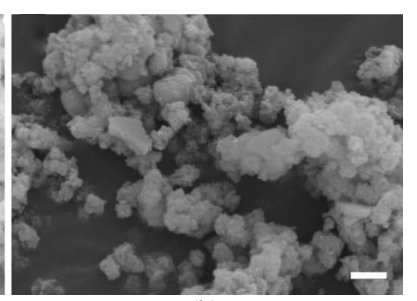

(b)

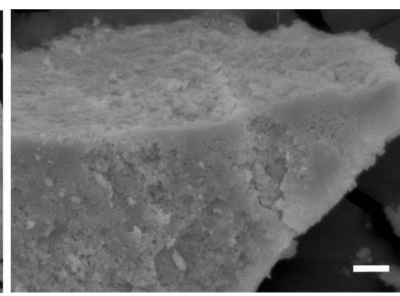

(d)

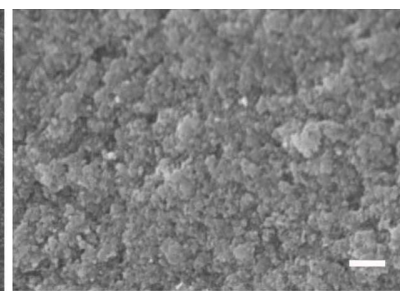

(f)
Figure 4. SEM images of the powders obtained on increaseing gelatin concentration. (a) CaP; (b) CaP0.05; (c) CaP0.1; (d) CaP0.5; (e)-(f) CaP1; Bars: (a), (b), (c), (f) = 2 micron; (d), (e) = 5 micron).

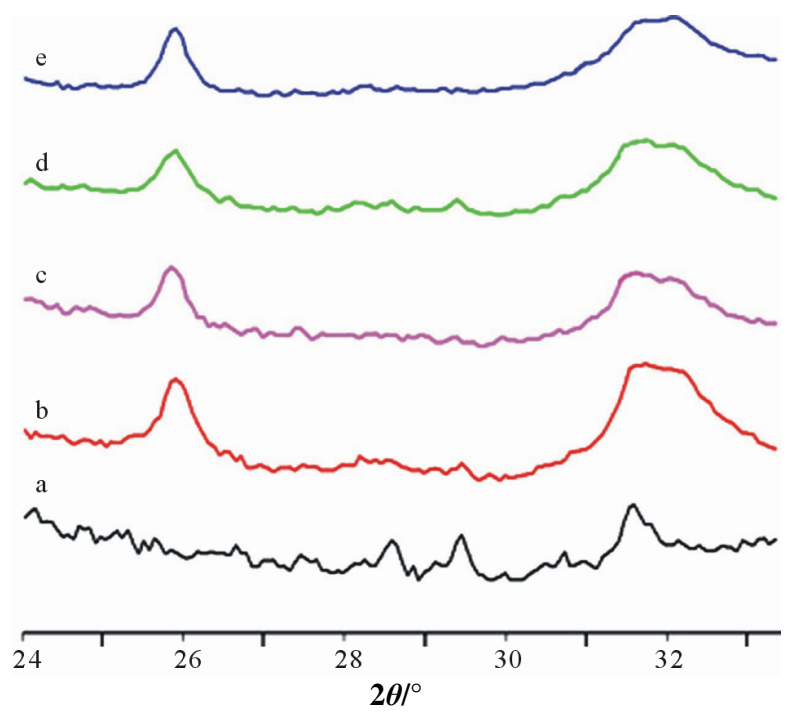

Figure 5. X-ray diffraction patterns recorded from the Ti substrates after $6 \mathrm{~h}$ soaking in the different calcifying solutions. (a) Ti substrate; (b) TiCaP; (c) TiCaP0.05; (d) TiCaP0.1; (e) TiCaP0.5.

calcifying solutions. The patterns indicate that immersion in the calcifying solutions provoked the deposition of an inorganic phase consisting of poorly crystalline apatite. The SEM micrographs of the Ti substrates after soaking are reported in Figure 6. TiCaP appears coated with a thin, but homogeneous, layer of inorganic deposition which is constituted of almost spherical aggregates, with mean dimensions of about $1 \mu \mathrm{m}$ (Figure 6(a)). Similar aggregates are present on $\mathrm{TiCaP} 0.05$ and $\mathrm{TiCaP} 0.1$ (Figures 6(b) and (c)), whereas the particles on $\mathrm{TiCaP} 0.5$ are even smaller (Figure 6(d)). The smaller dimensions of the spherical aggregates on the coatings (Figures 6(a) and (b)) with respect to those in the powders (Figures 4(a) and (b)) are coherent with the classical nucleation theory. The presence of a foreign surface, namely the Ti substrate, promotes nucleation and favours formation of a greater number of nuclei. As a consequence, nuclei growth is reduced with respect to that observed in the absence of the substrates. Furthermore, the inhibiting effect of gelatin provokes the decrease of the aggregates mean dimensions on increasing gelatin concentration up to $\mathrm{TiCaP1}$ where the particles are so small that they form a continuous layer which follows the substrate morphology (Figure 6(e)). The results obtained on the samples prepared in the presence of fluorescein dye confirm the presence of gelatin in the coatings. Figure 6(f) provides confocal microscopy images of the sample TiCaP01, containing fluorescent marker: gelatin is present and is homogeneously distributed in the coating.

\subsection{In Vitro Study}

In vitro tests were performed on coated substrates at two 


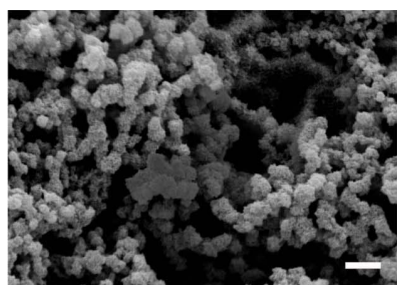

(a)

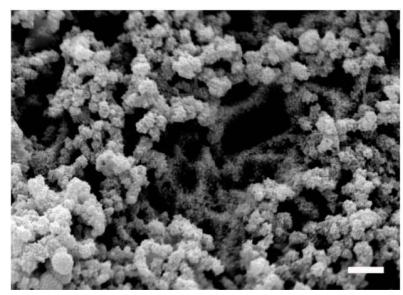

(c)

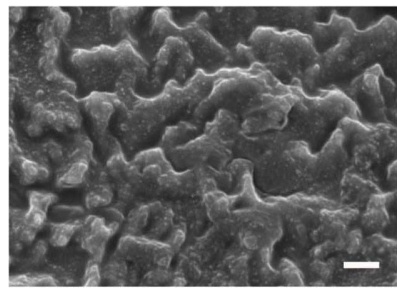

(e)

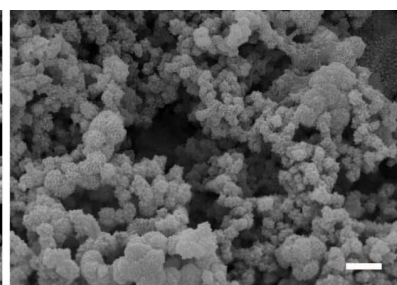

(b)

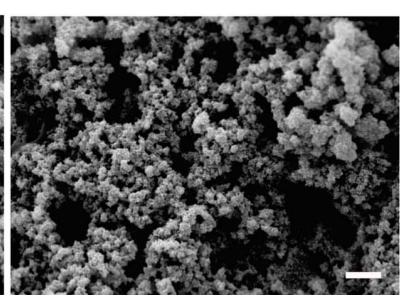

(d)

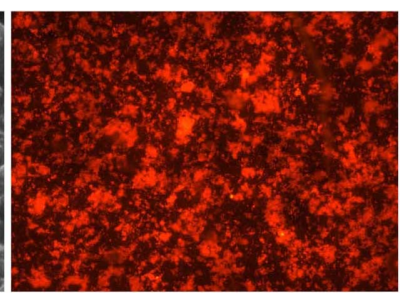

(f)
Figure 6. 6(a)-(e) SEM images of the coatings deposited on the Ti substrates after $6 \mathrm{~h}$ soaking in the different calcifying solutions. (a) TiCaP; (b) TiCaP0.05; (c) TiCaP0.1; (d) TiCaP0.5; (e) TiCaP1); (f) Confocal fluorescence microscope image of TiCaP0.1 with the addition of FITC.

different gelatin concentrations, TiCaP0.1 and TiCaP0.5, as well as on $\mathrm{TiCaP}$ as reference material.

\section{Osteoblast Proliferation and Activity}

MG63 osteoblast-like cell proliferation, activity and differentiation when cultured on TiCaP0.1 and TiCaP0.5 coatings were evaluated after 7 days of culture, and compared to TiCaP and CTR groups. After 24 hours from seeding no differences were found in cell proliferation among all groups (Figure 7(a)), as also shown by phalloidin staining (Figures 8(a)-(c)). The percent coverage of sample area by cells seeded and adhered to the surface after 24 hours of culture was calculated as the mean of ten fields for each sample and is reported in Figure 8. Cells adhered uniformly on all material samples, as on polystyrene (CTR). Osteoblast showed a regular shape and no differences were found in percent coverage. After 3 days of culture WST1 values of TiCaP and TiCaP0.1 were significantly higher than those of TiCaP0.5 and CTR, while at 7 days all experimental groups were significantly higher than CTR, demonstrateing that the TiCaP coatings enhance cell proliferation.

The evaluation of osteoblast differentiation was performed according to well-established parameters of osteoblastic phenotype: ALP, a specific indicator of os- teoblast activity is known to increase in the first phase of cell proliferation; COL I and OC, the major components of extracellular bone matrix, are synthesized in the following phase of differentiation and mineralization.

The results showed that the addition of gelatin to TiCaP coating significantly improved osteoblast activity, as reported in Figures 7(b)-(d) for ALP, COL I, and OC. ALP activity at 3 days was significantly higher in both TiCaP0.1 and TiCaP0.5 when compared to $\mathrm{TiCaP}$ and CTR groups (Figure 7(b)). COL I production on $\mathrm{TiCaP}$ was significantly higher than CTR group, but lower than TiCaP0.1 and TiCaP0.5 that reached the highest levels both at 3 and 7 days of culture (Figure 7(c)). OC synthesis was significantly higher on $\mathrm{TiCaP} 0.5$ with respect of all other groups already at 3 days, while at 7 days all the experimental groups showed significantly higher values in comparison with CTR: in particular TiCaP0.1 OC level was the highest, followed by $\mathrm{TiCaP} 0.5$ value, as shown in Figure 7(d).

TGFb1 is a growth factor implicated in modulation of cell proliferation and deposition of extracellular bone matrix, promoting synthesis and inhibiting degradation. The results of this study showed that TGFb1 activation significantly increased from day 3 to 7 in all groups (Figure 7(e)). In particular, $\mathrm{TiCaP}$ and $\mathrm{TiCaP0} .1$ displayed higher levels than the other groups. Moreover, TGFb1 activity was directly correlated with cell proliferation, collagen type I and osteocalcin production (Pearson correlation test, $\mathrm{p}<0.001$ ), while no correlation was found between TGFb1 and ALP activity.

Interleukin 6 (IL-6) is a pro-inflammatory cytokine, that has a major role in the mediation of inflammation, but it is also implicated in bone remodeling, since it stimulates the first stages of osteoblast differentiation. At 3 days its production was significantly higher on $\mathrm{TiCaP}$ and TiCaP0.1 than on the other groups, but at 7 days both values were reduced and no differences were found among the different groups (Figure 7(f)).

SEM analysis of MG63 was performed to evaluate morphology of cells grown on the different material surfaces after 7 days of culture. The osteoblasts were observed to attach and spread on coated surfaces. The cells generally appear well flattened and rich of filopodia, as shown in Figures 9(a) and (b) for $\mathrm{TiCaP}$ and $\mathrm{TiCaP} 0.1$.

To resume, all the coatings greatly influenced osteoblast behavior, as demonstrated by cell proliferation rate, COL I and OC production. The enrichment of the coating with gelatin significantly improved osteoblast proliferation and differentiation and yielded higher values of proliferation, COL I and OC production, as well as ALP activity stimulation. Although the levels of the differentiation parameters show minor differences between TiCaP0.1 and TiCaP0.5, the data indicate that both gelatin concentrations promote osteoblast proliferation and 
24 h $\square 3$ days $\square$ days
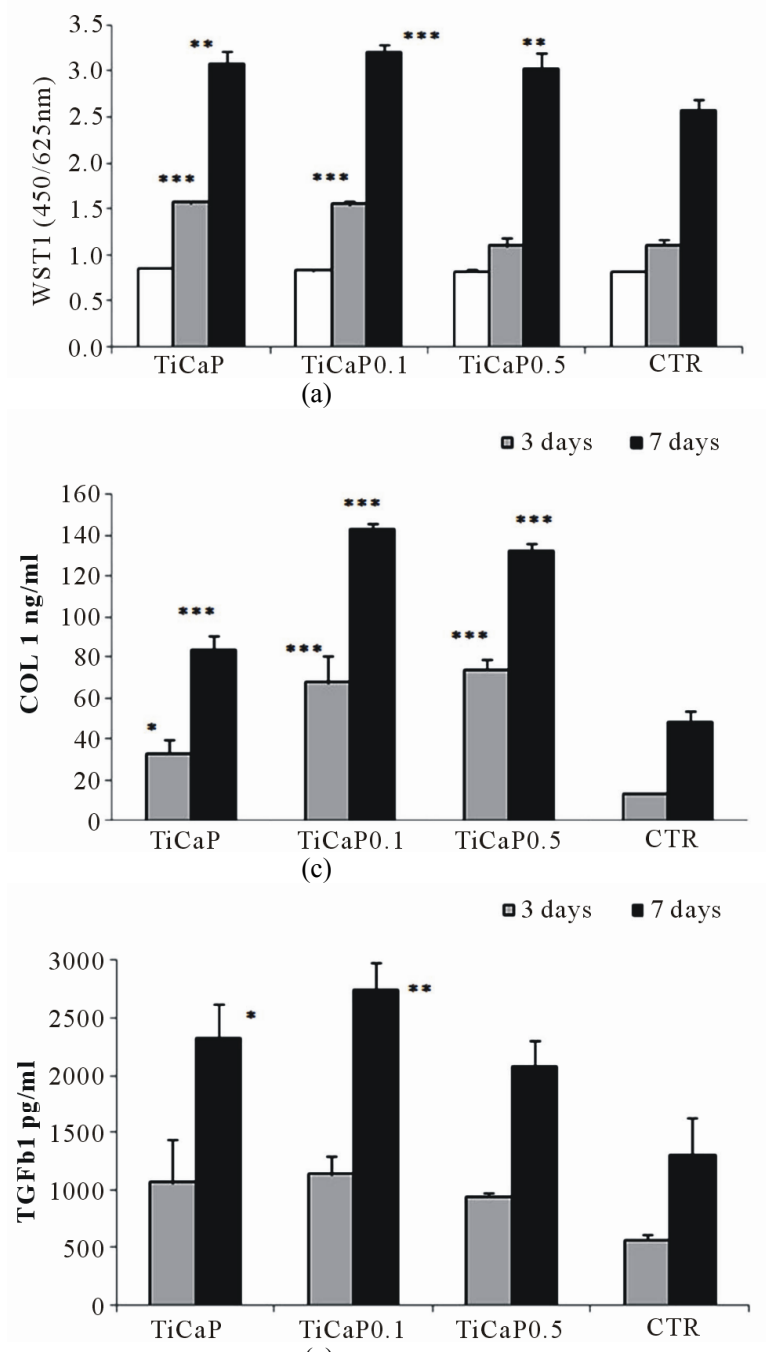

(e)

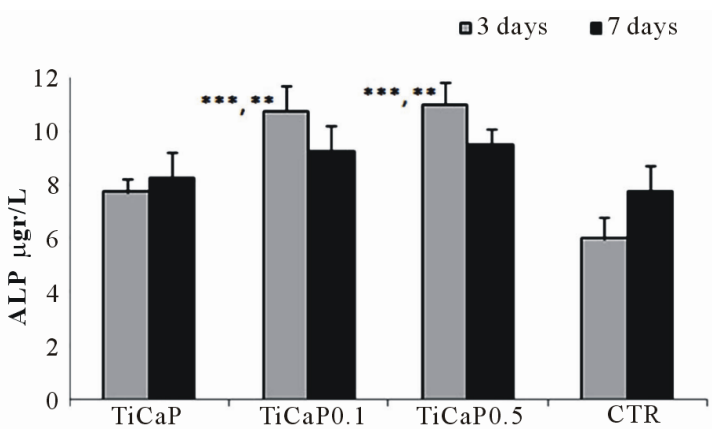

(b)
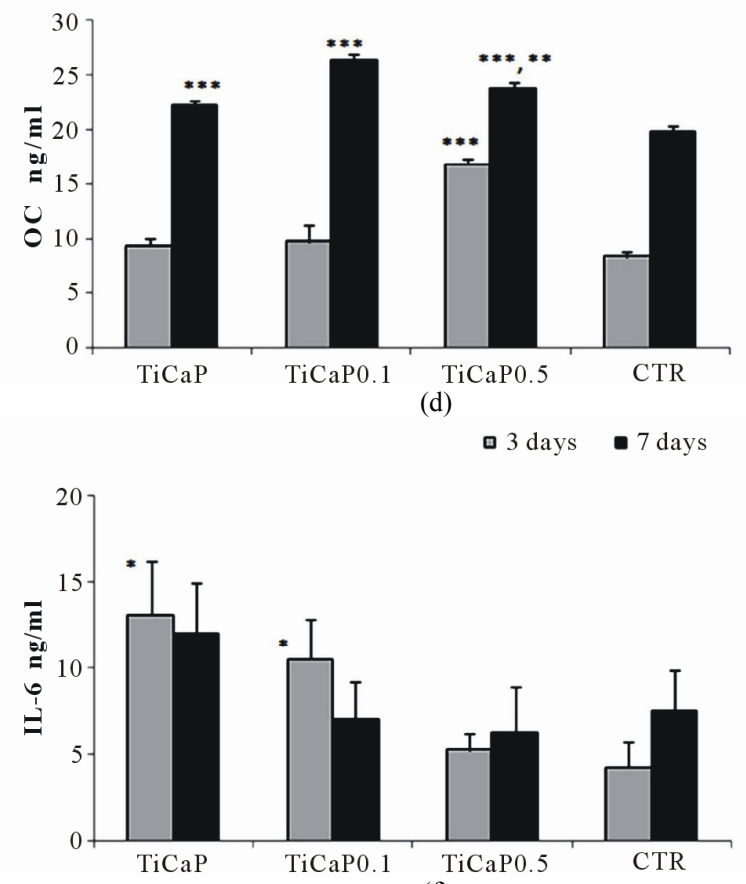

(f)

Figure 7. Proliferation, differentiation and synthetic activity of osteoblasts after culture on samples of TiCaP, TiCaP0.1, TiCaP0.5, and CTR. Mean \pm sd, $\mathbf{n}=6 .\left(^{*}=\mathbf{p}<0.05 ;{ }^{* *}=\mathbf{p}<0.005 ;{ }^{* * *}=\mathbf{p}<0.0001\right)$. (a) WST1. 3 days: ${ }^{* * *}$ TiCaP, TiCaP0.1 vs TiCaP0.5, CTR; 7 days: ${ }^{* *}$ TiCaP, Ti CaP0.5 vs CTR; ${ }^{* * *}$ TiCaP0.1 vs CTR. (b) ALP. 3 days: ${ }^{* *}$ TiCaP0.1, TiCaP0.5 vs TiCaP; ${ }^{* * *}$ TiCaP0.1, TiCaP0.5 vs CTR. (c) COL I. 3 days: ${ }^{* * *}$ TiCaP0.1, TiCaP0.5 vs TiCaP, CTR; ${ }^{*}$ TiCaP vs CTR; 7 days: ${ }^{* * *}$ TiCaP0.1, TiCaP0.5 vs TiCaP, CTR; ${ }^{* * *}$ TiCaP vs CTR. (d) OC. 3 days: ${ }^{* * *}$ TiCaP0.5 vs TiCaP, TiCaP0.1, CTR; 7 days: ${ }^{* * *}$ TiCaP vs CTR; ${ }^{* * *}$ TiCaP0.1 vs TiCaP, TiCaP0.5, CTR; ${ }^{* *}$ TiCaP0.5 vs TiCaP; ${ }^{* * *}$ TiCaP0.5 vs CTR. (e) TGFb1. 7 days: ${ }^{*}$ TiCaP vs CTR; ${ }^{* *}$ TiCaP0.1 vs CTR. (f) IL-6. 3days: ${ }^{* * *}$ TiCaP vs TiCaP0.1, TiCaP0.5, CTR; ${ }^{* *}$ TiCaP0.1 vs TiCaP0.5, CTR.

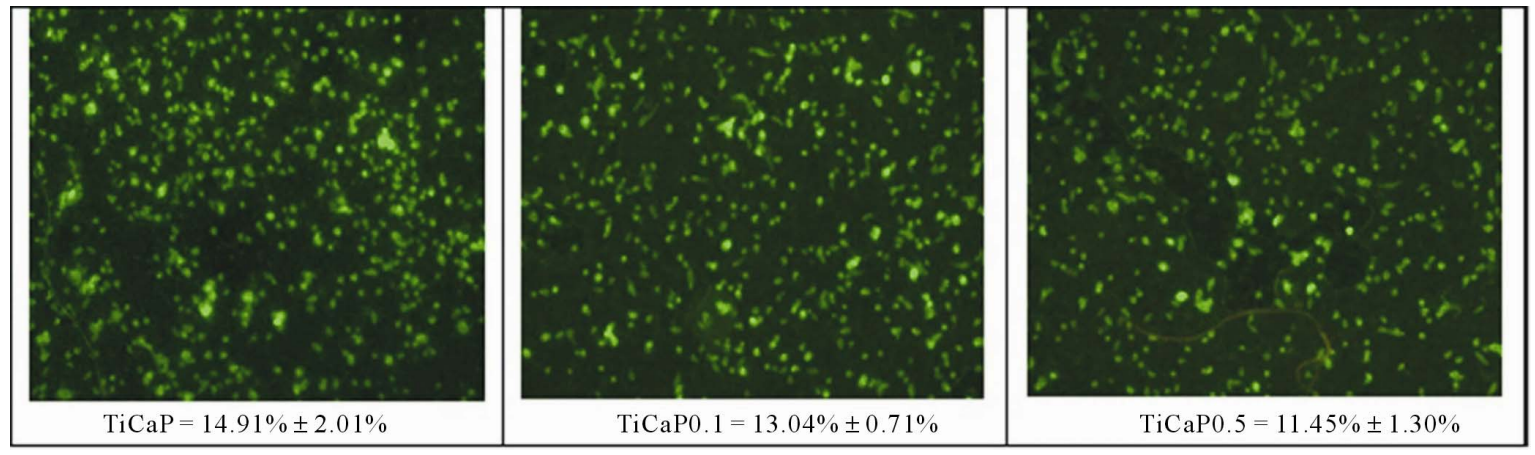

Figure 8. Phalloidin staining and percent coverage of sample area to assess cell adhesion and colonization of MG63 grown on TiCaP, TiCaP0.1, TiCaP0.5, after $24 \mathrm{~h}$ of culture. 


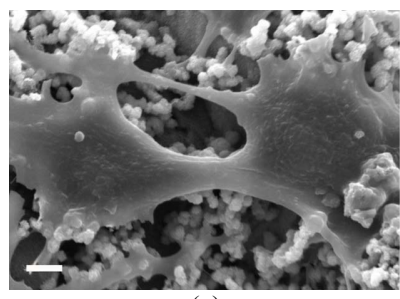

(a)

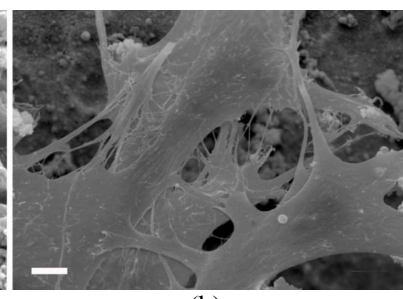

(b)
Figure 9. SEM images of MG63 cells after 7 days of culture onto (a) TiCaP and (b) TiCaP0.1 substrates.

differentiation.

\section{Conclusion}

The slightly supersaturated CaP solution can be successfully employed to get a fast deposition of thin gelatinapatite composite films on metallic substrates. Gelatin inhibits the crystallization of the inorganic phase: the dimensions of the spherical aggregates and the degree of crystallinity of the apatitic phase decrease on increasing gelatin concentration. The presence of gelatin improves osteoblast proliferation and differentiation, suggesting a beneficial role of the protein on bone cell response to the coatings.

\section{Acknowledgements}

This research was carried out with the financial support of MIUR.

\section{REFERENCES}

[1] S. R. Paital and N. B. Dahotre, "Calcium phosphate Coatings for Bio-Implant Applications: Materials, Performance Factors, and Methodologies," Materials Science and Engineering, Vol. 66, No. 1-3, 2009, pp. 1-70. doi:10.1016/j.mser.2009.05.001

[2] I. N. Mihailescu, C. Ristoscu, A. Bigi and I. Mayer. "Advanced Biomimetic Implants Based on Nanostructured Coatings Synthesized by Pulsed Laser Technologies," In: A. Miotello and P. M. Ossi, Laser-Surface Interactions for New Materials Production: Tailoring Structure and Properties, Springer, Berlin, 2009, pp. 235-260.

[3] A. W. G. Nijhuis, S. C. G. Leeuwenburgh and J. A. Jansen, "Wet-Chemical Deposition of Functional Coatings for Bone Implantology," Macromolecular Bioscience, Vol. 10, No. 11, 2010, pp. 1316-1329. doi:10.1002/mabi.201000142

[4] E. Boanini, M. Gazzano and A. Bigi, "Ionic Substitutions in Calcium Phosphates Synthesized at Low Temperature," Acta Biomaterialia, Vol. 6, No. 6, 2010, pp. 18821894.doi:10.1016/j.actbio.2009.12.041

[5] T. Kokubo, H. Kushitani, S. Sakka, T. Kitsugi and T. Yamamuro, "Solutions Able to Reproduce in Vivo Surface-Structure Changes in Bioactive Glass-Ceramic A$\mathrm{W}^{3}$," Journal of Biomedical Materials Research, Vol. 24,
No. 6, 1990, pp. 721-734. doi:10.1002/jbm. 820240607

[6] A. Bigi, E. Boanini, B. Bracci, A. Facchini, S. Panzavolta, F. Segatti and L. Sturba "Nanocrystalline Hydroxyapatite Coatings on Titanium: A New Fast Biomimetic Method," Biomaterials, Vol. 26, No. 19, 2005, pp. 4085-4089. doi:10.1016/j.biomaterials.2004.10.034

[7] Y. Liu, G. Wu and K. de Groot, "Biomimetic Coatings for Bone Tissue Engineering Of Critical-Sized Defects," Journal of the Royal Society Interface, Vol. 7, No. S5, 2010, pp. S631-S647. doi:10.1098/rsif.2010.0115.focus

[8] A. Pasinli, M. Yuksel, E. Celik, S. Sener and A. C. Tas, "A New Approach in Biomimetic Synthesis of Calcium Phosphate Coatings Using Lactic Acid-Na Lactate Buffered Body Fluid Solution," Acta Biomaterialia, Vol. 6, No. 6, 2010, pp. 2282-2288. doi:10.1016/j.actbio.2009.12.013

[9] Z. Wu, B. Feng, J. Weng, S. Qu, J. Wang and X. Lu, "Biomimetic Apatite Coatings on Titanium Coprecipitated with Cephradine and salviae miltlorrhizae," Journal of Biomedical Materials Research Part B: Applied Biomaterials, Vol. 84B, No. 2, 2008, pp. 486-492. doi: $10.1002 / \mathrm{jbm} . \mathrm{b} .30895$

[10] F. Barrere, C. M. van der Valk, G. Meijer, R. A. J. Dalmeijer, K. de Groot and P. Layrolle, "Osteointegration of Biomimetic Apatite Coating Applied onto Dense and Porous Metal Implants in Femurs of Goats," Journal of Biomedical Materials Research Part B: Applied Biomaterials, Vol. 67B, No. 1, 2003, pp. 655-665. doi:10.1002/jbm.b.10057

[11] A. L. Oliveira, R. L. Reis and P. Li, "Strontium-Substituted Apatite Coating Grown on Ti6Al4V Substrate through Biomimetic Synthesis," Journal of Biomedical Materials Research Part B: Applied Biomaterials, Vol. 83B, No. 1, 2007, pp. 258-265. doi:10.1002/jbm.b.30791

[12] B. Bracci, P. Torricelli, S. Panzavolta, E. Boanini, R. Giardino and A. Bigi, "Effect of $\mathrm{Mg}^{2+}, \mathrm{Sr}^{2+}$, and $\mathrm{Mn}^{2+}$ on the Chemico-Physical and in Vitro Biological Properties of Calcium Phosphate Biomimetic Coatings," Journal of Inorganic Biochemistry, Vol. 103, No. 12, 2009, pp. 16661674. doi:10.1016/j.jinorgbio.2009.09.009

[13] C. Chen, I. S. Lee, S. M. Zhang and H. C. Yang, "Biomimetic Apatite Formation on Calcium Phosphate-Coated Titanium in Dulbecco's Phosphate-Buffered Saline Solution Containing $\mathrm{CaCl}_{2}$ with and without Fibronectin," Acta Biomaterialia, Vol. 6, No. 6, 2010, pp. 2274-2281. doi:10.1016/j.actbio.2009.11.033

[14] Y. Liu, P. Layrolle, J. de Bruijn, C. van Blitterswijk and K. de Groot, "Biomimetic Coprecipitation of Calcium Phosphate and Bovine Serum Albumin on Titanium Alloy", Journal of Biomedical Materials Research, Vol. 57, No. 3, 2001, pp. 327-335. doi:10.1002/1097-4636(20011205)57:3<327::AID-JBM1 $175>3.0 . \mathrm{CO} ; 2-\mathrm{J}$

[15] Y. Liu, J. P. Li, E. B. Hunziker and K. de Groot, "Incor-Poration of Growth Factors into Medical Devices via Biomimetic Coatings," Philosophical Transactions of the Royal Society A, Vol. 364, No. 1838, 2006, pp. 233-248. 
doi:10.1098/rsta.2005.1685

[16] L. N. Luong, S. I. Hong, R. J. Patel, M. E. Outslay and D. H. Kohn "Spatial Control of Protein within Biomimetically Nucleated Mineral," Biomaterials, Vol. 27, No. 7, 2006, pp. 1175-1186. doi:10.1016/j.biomaterials.2005.07.043

[17] X. Yu, H. Qu, D. A. Knecht and M. Wei, "Incorporation of Bovine Serum Albumin into Biomimetic Coatings on Titanium with High Loading Efficacy and Its Release Behavior," Journal of Materials Science: Materials in Medicine, Vol. 20, No. 1, 2009, pp. 287-294. doi:10.1007/s10856-008-3571-6

[18] Y. Chen, A. F. T. Mak, M. Wang, J. S. Li and M. S. Wong, "In Vitro Behavior of Osteoblast-Like Cells on PLLA Films with a Biomimetic Apatite or Apatite/Collagen Composite Coating," Journal of Materials Science: Materials in Medicine, Vol. 19, No. 6, 2008, pp. 22612268. doi:10.1007/s10856-007-3335-8

[19] K. Hu, X. J. Yang, Y. L. Cai, Z. D. Cui, Q. Wei, "Preparation of Bone-Like Composite Coating Using a Modified Simulated Body Fluid with high $\mathrm{Ca}$ and $\mathrm{P}$ Concentrations," Surface and Coatings Technology, Vol. 201, No. 3-4, 2006, pp. 1902-1906. doi:10.1016/j.surfcoat.2006.02.036

[20] S. Lee, D. Suarez-Gonzales and W. Murphy, "Mineral Coatings for Temporally Controlled Delivery of Multiple Proteins," Advanced Materials, Vol. 23, No. 37, 2011, pp. 4279-4284. doi:10.1002/adma.201100060

[21] X. J. Yang, C. Y. Liang, Y. L. Cai, K. Hu, Q. Wei and Z. D. Cui, "Recombinant Human-Like Collagen Modulated the Growth of Nano-Hydroxyapatite on NiTi alloy," $M a$ terials Science and Engineering C, Vol. 29, No. 1, 2009, pp. 25-28. doi:10.1016/j.msec.2008.05.006

[22] A. Bigi, S. Panzavolta and K. Rubini, "Relationship Between Triple Helix Content and Mechanical Properties of Gelatin Films," Biomaterials, Vol. 25, No. 25, 2004, pp. 5675-5680. doi:10.1016/j.biomaterials.2004.01.033

[23] G. Tronci, A. T. Neffe, B. F. Pierce and A. Lendlein, "An Entropy- Elastic Gelatin-Based Hydrogel System," Jour- nal of Materials Chemistry, Vol. 20, No. 40, 2010, pp. 8875-8884. doi:10.1039/c0jm00883d

[24] J. F. Mano, "Natural Origin Biodegradable Systems in Tissue Engineering and Regenerative Medicine: Present Status and Some Moving Trends," Journal of the Royal Society Interface, Vol. 4, No. 17, 2007, pp. 999-1030. doi:10.1098/rsif.2007.0220

[25] A. Bigi, N. Nicoli-Aldini, B. Bracci, B. Zavan, E. Boanini, F. Sbaiz, S. Panzavolta, G. Zorzato, R. Giardino, A. Facchini, G. Abatangelo and R. Cortivo, "In Vitro Culture of Mesenchymal Cells onto Nanocrystalline Hydroxyapatite-Coated Ti13Nb13Zr Alloy," Journal of Biomedical Materials Research Part A, Vol. 82, No. 1, 2007, pp. 213221. doi:10.1002/jbm.a.31132

[26] R. Chiesa, E. Sandrini, M. Santin, E. Rondelli and A. Cigada, "Osteointegration of Titanium and Its Alloys by Anodic Spark Deposition and other Electrochemical Tecniques: A Review," Journal of Applied Biomaterials and Biome- chanic, Vol. 1, No. 2, 2003, pp. 91-107.

[27] A. Bigi, G. Cojazzi, M. H. J. Koch, G. Pizzuto, A. Ripamonti and N. Roveri, "Structural Analysis of Turkey Tendon Collagen upon Removal of the Inorganic Phase," International Journal of Biological Macromolecules, Vol. 13, No. 2, 1991, pp. 110-114. doi:10.1016/0141-8130(91)90058-3

[28] S. Busch, U. Schwarz and R. Kniep, "Morphogenesis and Structure of Human Teeth in Relation to Biomimetically Grown Fluorapatite-Gelatin Composites," Chemistry Materials, Vol. 13, No. 10, 2001, pp. 3260-3271. doi:10.1021/cm0110728

[29] M. Iijima, Y. Moriwaki, T. Takagi and J. MoradianOldak, "Effects of Bovine Amelogenins on the Crystal Morphology of Octacalcium Phosphate in a Model System of Tooth Enamel Formation," Journal of Crystal Growth, Vol. 222, No. 3, 2001, pp. 615-626. doi:10.1016/S0022-0248(00)00984-2

[30] A. Bigi, S. Panzavolta and K. Rubini, "Setting Mechanism of a Biomimetic Bone Cement," Chemistry Materials, Vol. 16, No. 19, 2004, pp. 3740-3745. doi: $10.1021 / \mathrm{cm} 049363 \mathrm{e}$ 